\title{
Sulodexide: implicazioni cliniche ed economiche
}

Orietta Zaniolo*, Mario Eandi§

REVIEW

\begin{abstract}
Sulodexide is a highly purified glycosaminoglycan approved for leg ulcers treatment. It contains two principal components: heparan sulfate, a fast-moving heparin fraction, (80\%) and dermatan sulfate (20\%).

Sulodexide is available as an oral agent and as an injectable preparation.

Its pharmacological action is obtained by dose-dependent coagulation factors inhibition: dermatan sulfate upgrades the physiological action of a selective thrombin inhibitor, heparin cofactor II, and heparan sulfate depresses activated factor X, via an increase of antithrombin III action.

The antithrombotic action is enhanced by platelet aggregation inhibition and by the activation of the fibrinolytic system.
\end{abstract}

This paper summarizes the results of some of the main trials that evaluated sulodexide in the treatment of peripheral occlusive arterial disease and venous leg ulcers; a trial on prevention of recurrent deep venous thrombosis with sulodexide is also reviewed.

We analyzed data about the clinical and economical impact of chronic venous insufficiency with a particular attention to the cost of medication, hospitalization and management of leg ulcers.

The hypothetical savings correlated to the reduction of leg ulcers incidence and healing time attainable with sulodexide have been estimated. A comparison between the different acquisition costs of the drugs frequently used to treat leg ulcers is also provided.

Finally we reviewed some quality of life trials in which the psychological and sociological influence of the disease and its treatments on the patient are assessed.

Keywords: sulodexide, chronic venous insufficiency, leg ulcers, cost of illness Farmeconomia e percorsi terapeutici 2005; 6 (2): 105-118

\section{INTRODUZIONE}

Sulodexide è un farmaco antitrombotico eparinico costituito da una miscela di glicosaminoglicani (GAGs) estratti da mucosa intestinale suina, costituita da una frazione eparinica a medio peso molecolare e da dermatan solfato (DS), un glicosaminoglicano chimicamente distinto dall'eparina e derivati per la presenza di galattosamina.

Scopo di questo lavoro è delineare un profilo farmacologico clinico e farmacoeconomico del farmaco, analizzando in modo particolare la sua efficacia nel trattamento di pazienti affetti da forme croniche di Insufficienza Venosa (ulcere venose croniche), nella prevenzione delle recidive di trombosi venosa profonda e nella cura dell' arteriopatia ostruttiva periferica.

\section{FARMACOLOGIA}

\section{Farmacodinamica}

In caso di lesione della parete di un vaso le cellule endoteliali espongono il tessuto connettivo sottoendoteliale altamente trombogenico, al quale le piastrine aderiscono entrando in uno stato di "attivazione" che induce in loro un cambiamento morfologico-strutturale e una reazione di esocitosi.

I fattori che si liberano dai granuli piastrinici (ADP, $\mathrm{TXA}_{2}$, serotonina e altri) stimolano il meccanismo di auto-amplificazione della risposta piastrinica così da formare il tappo piastrinico. Tale reazione avviene entro pochi minuti dalla lesione e, insieme alla vasocostrizione, costituisce la cosiddetta emostasi primaria.

\section{* Advanced Research \\ $\S$ Farmacologia Clinica Università di Torino}


Il sistema della coagulazione è il componente successivo del processo emostatico e porta alla formazione del coagulo insolubile di fibrina.

Tutti i fattori della coagulazione, ad eccezione del fattore III (fattore tessutale), sono normalmente presenti nel plasma in forma inattiva e vengono attivati mediante l'esposizione di superficie negativa (meccanismo intrinseco) e di fattore tessutale (meccanismo estrinseco) nel sito di lesione; secondo le teorie più recenti l'intervento più rilevante è quello del fattore tessutale (TF), il quale ha affinità per una proteina plasmatica (il fattore VII), e questo porta alla formazione di un complesso TF-FVIIa che ha la capacità non solo di attivare direttamente il FX a FXa e quindi proseguire nella via comune (classica via estrinseca), ma anche il FIX della via intrinseca (cross-over).

Il fattore $\mathrm{X}$ attivato trasforma la protrombina in trombina circolante, che converte il fibrinogeno in fibrina, formando un coagulo insolubile e stimolando un ulteriore reclutamento di piastrine.

Una volta che la lesione vascolare è stata riparata, si verifica la dissoluzione del coagulo stesso mediante il processo di fibrinolisi, il cui evento centrale consiste nella trasformazione del plasminogeno inattivo in plasmina; questo enzima proteolitico svolge un'azione di rimodellamento del trombo e ne limita l'estensione attraverso la digestione proteolitica della fibrina.

Affinché non si abbia l'estensione del coagulo nel sistema vascolare, è necessario che il processo della coagulazione sia finemente controllato e circoscritto nel punto di lesione.

Esistono vari meccanismi di controllo:

- il flusso sanguigno è fondamentale nel controllo dell' emostasi in quanto è responsabile dell'allontanamento e della diluizione dei fattori della coagulazione attivati, che vengono poi rimossi dal circolo;

- un altro tipo di controllo altrettanto importante è rappresentato dall'inattivazione di fattori e cofattori della coagulazione da parte degli inibitori fisiologici.

Queste sostanze, in alcuni casi, possono inibire sia gli enzimi del sistema della coagulazione che quelli del sistema fibrinolitico: si tratta quindi di un sistema di controllo che è in grado di impedire sia una eccessiva amplificazione e propagazione del coagulo sia un'eccessiva attivazione del sistema fibrinolitico che potrebbe portare a manifestazioni di tipo emorragico.

Gli inibitori fisiologici più importanti sono l'Antitrombina-III (AT-III), il Cofattore eparinico II (HCII), le proteine $C$ e $S$, l'inibitore della via del fattore tessutale (TFPI).

La trombosi può essere vista come un'estensione patologica del normale meccanismo emostatico: alterazioni locali e generali del flusso sanguigno e dei componenti ematici dell'emostasi impediscono la normale dissoluzione del coagulo con formazione del trombo.

Sulodexide è una miscela di glucosaminoglicani strutturalmente simile ai derivati eparinici a basso peso molecolare costituita per 1' $80 \%$ da eparina a medio peso molecolare e per il $20 \%$ da dermatan solfato; il suo peso molecolare medio è di circa $8000 \mathrm{Da}[1,2]$.

La sua azione antitrombotica è dovuta all'inibizione dose-dipendente di alcuni fattori coagulativi: il dermatan solfato potenzia l'azione di un inibitore fisiologico selettivo della trombina, il cofattore eparinico II, mentre la frazione eparinica inibisce il fattore $\mathrm{X}$ attivato, potenziando l'azione dell' antitrombina III.

Sulodexide prolunga il Tempo di Trombina (TT) in maniera dose-dipendente, fino ad impedire completamente la coagulazione nel momento in cui la concentrazione plasmatica supera il valore di $2 \mathrm{mg} / \mathrm{ml}$ [3]; esso è in grado di prolungare anche il Tempo di Tromboplastina Parziale attivata (aPTT) [4].

L'azione antitrombotica è sostenuta anche dall'inibizione dell' adesività piastrinica e dall'attivazione del sistema fibrinolitico: esso stimola infatti il rilascio dell' attivatore tissutale del plasminogeno e impedisce l' azione del suo inibitore (PAI-1) [2].

Sulodexide è in grado di normalizzare i parametri viscosimetrici che di solito risultano alterati in pazienti con patologie vascolari a rischio trombotico: tale attività si esercita principalmente mediante la riduzione dei livelli plasmatici di fibrinogeno; inoltre il farmaco, mediante attivazione della lipoproteinlipasi, contribuisce alla normalizzazione dei valori lipidici alterati [5].

Il farmaco ha dimostrato proprietà antitrombotiche e profibrinolitiche in seguito a somministrazione orale, subcutanea e intravenosa, manifestando un'elevata affinità per le cellule endoteliali.

\section{Farmacocinetica}

Sulodexide è commercializzato in capsule molli e in soluzione iniettabile; capsule e fiale contengono rispettivamente 250 ULS (unità lipasemiche) e 600 ULS ognuna. Un milligrammo di farmaco equivale a 10 unità lipasemiche.

Prove farmacologiche eseguite nell'uomo in seguito a somministrazione i.m. e i.v. di sulodexide hanno dimostrato relazioni lineari dose-effetto e un'elevata prevedibilità per 
quanto riguarda la dose assorbita dopo somministrazione.

Il farmaco ha dimostrato un elevato assorbimento attraverso la barriera gastrointestinale offrendo la possibilità di assunzione per via orale.

Il farmaco tende naturalmente a concentrarsi nell'endotelio; inizialmente il sulodexide marcato si accumula nelle cellule dell'intestino per poi essere liberato nel circolo sistemico: la sua concentrazione aumenta nel tempo a livello di cervello, rene, cuore, fegato, polmone, testicolo e plasma.

Nell'uomo in seguito a somministrazione orale si osserva un primo picco ematico dopo circa 2 ore e un secondo picco tra la quarta e le sesta ora, dopodiché il farmaco non è più riscontrabile nel plasma; esso ricompare verso la dodicesima ora, rimanendo quindi costante fin verso la quarantottesima ora: tale valore costante è stato imputato al lento rilascio del farmaco da parte degli organi di captazione ed in particolare dell'endotelio dei vasi [5]. La sua emivita è di 11-26 ore.

Il metabolismo è principalmente epatico e l'escrezione principalmente urinaria.

\section{EFFICACIA TERAPEUTICA E TOLLERABILITÀ}

\section{Flebopatie derivanti da insufficienza venosa}

Il processo patologico all'origine dell'Insufficienza Venosa Cronica (IVC) può comportare quadri clinici e funzionali di diversa gravità. La classificazione CEAP (classificazione Clinica, Eziologica, Anatomica, Patofisiologica) è stata messa a punto da un gruppo internazionale di specialisti nel 1994 allo scopo di ottenere una nuova modalità standardizzata di valutazione delle flebopatie croniche [6]. Riportiamo in Tabella I la sezione clinica di tale classificazione.

L'incompetenza valvolare del circolo venoso profondo o superficiale costituisce spesso la causa di sviluppo di IVC: maggiore è il numero degli apparati valvolari lesi, maggiore sarà il reflusso venoso e come conseguenza si osserverà un aumento della pressione venosa deambulatoria.

L'incremento della pressione venosa, derivante dall' alterato ritorno venoso, può condurre al quadro della microangiopatia venosa ipertensiva (VHM - venous hypertensive microangiopathy), che è caratterizzata da un aumento della vasodilatazione cutanea, da un marcato incremento della $\mathrm{pCO}_{2}$, da un aumento della permeabilità capillare e da una riduzione dell'attività fibrinolitica che determina accumu-

\begin{tabular}{|c|c|}
\hline Classe 0 & Assenza di segni clinici visibili o palpabili di malattia venosa \\
\hline Classe 1 & Presenza di teleangiectasie o vene reticolari \\
\hline Classe 2 & Presenza di vene varicose \\
\hline Classe 3 & Presenza di edema \\
\hline Classe 4 & $\begin{array}{l}\text { Turbe trofiche di origina venosa: pigmentazione, eczema, } \\
\text { ipodermite }\end{array}$ \\
\hline Classe 5 & Come classe 4 con ulcere cicatrizzate \\
\hline Classe 6 & Come classe 4 con ulcere in fase attiva \\
\hline a & Asintomatico \\
\hline $\mathbf{s}$ & Sintomatico \\
\hline
\end{tabular}

Tabella I

Classificazione clinica (C 0-6) delle flebopatie croniche derivanti da insufficienza venosa

lo di fibrina intorno ai capillari più distali [7]. Il riflesso venoarteriolare (VAR) di origine simpatica determina una riduzione del flusso cutaneo nel passaggio dal clinostatismo all'ortostatismo; tale riflesso è ridotto nell'IVC a causa dell' elevata $\mathrm{pCO}_{2}$ cutanea che mantiene la vasodilatazione: l'edema della caviglia si correla inversamente all'efficacia del VAR, ed è proprio la riduzione di questo che favorirebbe l'aumento della filtrazione capillare e lo stravaso di fluidi dal compartimento capillare a quello extravasale.

La maggior parte delle ulcere venose si colloca al di sopra del malleolo mediale o, meno frequentemente, di quello laterale. Queste regioni della caviglia sono drenate da una rete di piccole tributarie delle vene profonde (VP) che normalmente non sono visibili. A seguito dell'insufficienza delle VP, tali venule si sovraccaricano, si dilatano e si gonfiano e danno luogo all'eritema venoso della caviglia, segno che può essere frequentemente osservato prima dello sviluppo della lipodermatosclerosi o dell'ulcerazione.

Le alterazioni cellulari, biochimiche e tessutali che portano all'ulcerazione non sono ancora del tutto chiare; le ipotesi sostenute fino ad ora prendono in considerazione diversi fattori, come gli shunt artero-venosi, la formazione di cuffie di fibrina, l'adesione leucocitaria, lo stress ossidativo, i quali potrebbero favorire il processo flogistico responsabile di continue microemorragie (dermatiti ocra), eczema, dermoipodermiti e infine ulcera.

Le ulcere tendono ad infettarsi e l'infezione ostacola la guarigione, che può richiedere anche mesi o anni. 


\section{Tabella II}

Risultati ottenuti dai due gruppi dopo 90 giorni di trattamento, riportati sia in base alla popolazione intent-to-treat e sia escludendo dal calcolo i pazienti che, per varie ragioni, non hanno fatto uso di bendaggi di compressione [9]

$\mathrm{Pz}=$ pazienti

$\mathrm{ns}=$ non significativo

\begin{tabular}{lcccc}
\hline & \multicolumn{2}{c}{ Pazienti Intent-to-treat } & \multicolumn{2}{c}{ Pazienti con bendaggio } \\
\hline Trattamento & Sulodexide & Placebo & Sulodexide & Placebo \\
Numero pazienti & 120 & 110 & 102 & 93 \\
\hline & RISULTATI dopo 90 giorni di trattamento & \\
$\begin{array}{l}\text { Pz con guarigione completa } \\
\text { entro 2 mesi di trattamento }\end{array}$ & $42(35,0 \%)$ & $23(23,9 \%)$ & $41(40,2 \%)$ & $22(23,7 \%)$ \\
$\begin{array}{l}\text { Pz con guarigione completa } \\
\text { entro 3 mesi di trattamento } \\
\text { Andamento tempo-area lesa }\end{array}$ & $63(52,5 \%)$ & $36(32,7 \%)$ & $60(58,8 \%)$ & $36(38,7 \%)$ \\
\hline
\end{tabular}

\section{Trattamento delle ulcere venose}

Un'ulcera venosa (UV) su base cronica viene affrontata solitamente con medicazioni, terapia elastocompressiva e deambulazione; sfortunatamente in molti casi di lesione cronica degli arti inferiori questo approccio fallisce, con ulcere che rimangono aperte per mesi o anni.

Negli ultimi anni molti medici e ricercatori hanno affiancato ai metodi tradizionali diverse terapie sistemiche al fine di intervenire su alcuni processi biochimici e ristabilire l'equilibrio a livello di microcircolo [8].

Un trial multicentrico condotto da Coccheri e colleghi ha valutato l'efficacia di sulodexide, assunto in associazione con trattamenti topici e terapia elastocompressiva, nel favorire la guarigione dell'ulcera venosa.

I pazienti ammessi al trial dovevano essere di età compresa fra i 18 e i 75 anni e presentare ulcere croniche agli arti inferiori, di diametro non inferiore ai $2 \mathrm{~cm}$. Dei 230 partecipanti allo studio, 120 sono stati randomizzati in doppio cieco a ricevere sulodexide e 110 a ricevere placebo; il farmaco è stato somministrato per via intramuscolare nei primi 20 giorni $(1 \mathrm{f}=60$ $\mathrm{mg} / \mathrm{die}$ ) e per via orale nei successivi 70 giorni (4 cps $=100 \mathrm{mg} / \mathrm{die})$.

L'outcome primario dello studio era la percentuale di ulcere completamente guarite entro due mesi di trattamento; gli outcome secondari erano la percentuale di ulcere completamente guarite entro tre mesi di trattamento e l'andamento temporale della riduzione dell' area lesa.

Il tasso di recesso è stato del $25,8 \%$ nel gruppo trattato e del $21,8 \%$ nel gruppo controllo; i principali risultati di tale studio sono riassunti nella tabella II.

Come si evince dalla tabella II, sulodexide è efficace nell' aumentare frequenza e velocità di guarigione rispetto a placebo e i risultati ottenuti in seguito a due mesi di trattamento sono stati confermati dai dati raccolti al termine dello studio (tre mesi); la differenza di efficacia tra i due trattamenti è risultata statisticamente significativa in entrambe le popolazioni considerate.
La riduzione dell' area lesa è risultata significativamente associata al tempo di trattamento con sulodexide; tale correlazione, misurata mediante analisi multifattoriale, non è risultata significativa nel caso di trattamento con placebo.

Per quanto riguarda la tollerabilità del farmaco, 37 pazienti in tutto hanno riportato 40 effetti collaterali: $23(19,1 \%)$ nel gruppo trattato e $17(15,4 \%)$ nel gruppo controllo.

Trentuno pazienti $(25,8 \%)$ riceventi sulodexide e $24(21,8 \%)$ riceventi placebo non hanno terminato il periodo di trattamento; in entrambi i casi 7 pazienti hanno abbandonato lo studio a causa della comparsa di eventi avversi.

Nel complesso il farmaco è stato ben tollerato e non sono state riportate differenze significative fra i due trattamenti per quanto concerne la comparsa di eventi avversi.

I dati ottenuti in questo studio concordano con quelli risultanti da uno studio precedente, in cui 94 pazienti furono randomizzati a ricevere, in aggiunta ad elastocompressione, sulodexide oppure placebo. Al termine dei 2 mesi di trattamento previsti dallo studio l'ulcera appariva completamente guarita nel $36 \%$ dei pazienti controllo e nel $58 \%$ dei pazienti trattati, per una differenza statisticamente significativa $(\mathrm{P}=0,03)$.

\section{Prevenzione di recidive di trombosi venosa profonda e superficiale}

Gli effetti del farmaco sono stati indagati anche per ciò che riguarda il trattamento della Sindrome Post-Trombotica (SPT) e la prevenzione della trombosi venosa profonda [10-13].

Oltre all'evento tromboembolico singolo, per il quale vi è largo consenso sull'utilizzo di eparina (solitamente a basso peso molecolare) seguita da una fase di profilassi secondaria con anticoagulante orale (TAO) [14], l'insufficienza venosa cronica può essere correlata anche a ripetuti eventi di trombosi venosa profonda (R-TVP) spesso asintomatici e che, per tale ragione, non vengono riconosciuti dal paziente come un nuovo evento. 
La necessità di un trattamento anticoagulante prolungato, inteso quale prevenzione dell'estensione della trombosi e delle recidive tromboemboliche è inequivocabilmente dimostrata $[15,16]$; gli orientamenti attuali prevedono la somministrazione contemporanea di eparina e farmaci anti-vitamina $\mathrm{k}$ come il warfarin. La complicanza più frequente durante la TAO è costituita dalle manifestazioni emorragiche [17].

La durata ottimale della TAO dopo un primo episodio di TVP è ancora oggetto di dibattito; secondo le principali linee guida, per pazienti con TVP associata ad un fattore di rischio reversibile, il trattamento dovrebbe essere abbastanza breve (3-6 mesi) [17].

Dopo questo periodo non vi è un piano terapeutico largamente riconosciuto per continuare a lungo termine la prevenzione di retrombosi e sindrome post-trombotica.

Lo scopo di un trial indipendente condotto nell'ambito del progetto "San Valentino" (progetto di studio della prevalenza e dell'evoluzione dell'arteriosclerosi subclinica e delle malattie vascolari in Italia centrale) è stato quello di valutare gli effetti preventivi del trattamento con sulodexide in pazienti a medio o alto rischio trombotico [13].

I 405 pazienti inclusi nello studio sono stati trattati inizialmente con EBPM e poi con anticoagulante orale per un periodo di sei mesi, dopodiché sono stati divisi random in due gruppi: uno ricevente sulodexide per via orale $(2 \mathrm{cps}=50 \mathrm{mg} / \mathrm{die})$ e uno ricevente placebo per un periodo di due anni; in entrambi i casi i pazienti hanno effettuato prevenzione con calze elastiche a bassa compressione.

Hanno terminato i 24 mesi di trattamento 189 pazienti trattati con sulodexide $($ ITT $=203)$ e 178 trattati con placebo $($ ITT $=202)$.

Dopo 6 e 12 mesi il tasso d'incidenza delle retrombosi era significativamente minore nel gruppo trattato; a 24 mesi l'incidenza globale era di $17,9 \%$ nel gruppo controllo e $7,4 \%$ fra i pazienti trattati, 2,42 volte in meno (Figura 1).

Si sono verificati 32 TVP fra i pazienti controllo e 14 nel gruppo trattato; le trombosi superficiali riportate sono state 22 nel gruppo controllo e 8 nel gruppo trattato (di cui rispettivamente 18 e 6 concomitanti alla TVP) (Figura 2).

All'inizio del trial la localizzazione delle trombosi nei due gruppi era paragonabile; dopo 24 mesi di trattamento con sulodexide tutte le retrombosi distali risultavano più piccole $\mathrm{e}$ posizionate inferiormente rispetto a quelle presenti nei pazienti controllo.

Alla fine del trattamento, l'aumento del valore medio di D-dimero era stato contenuto entro il 2,6\% fra i pazienti trattati contro il 7,8\% dei pazienti controllo.

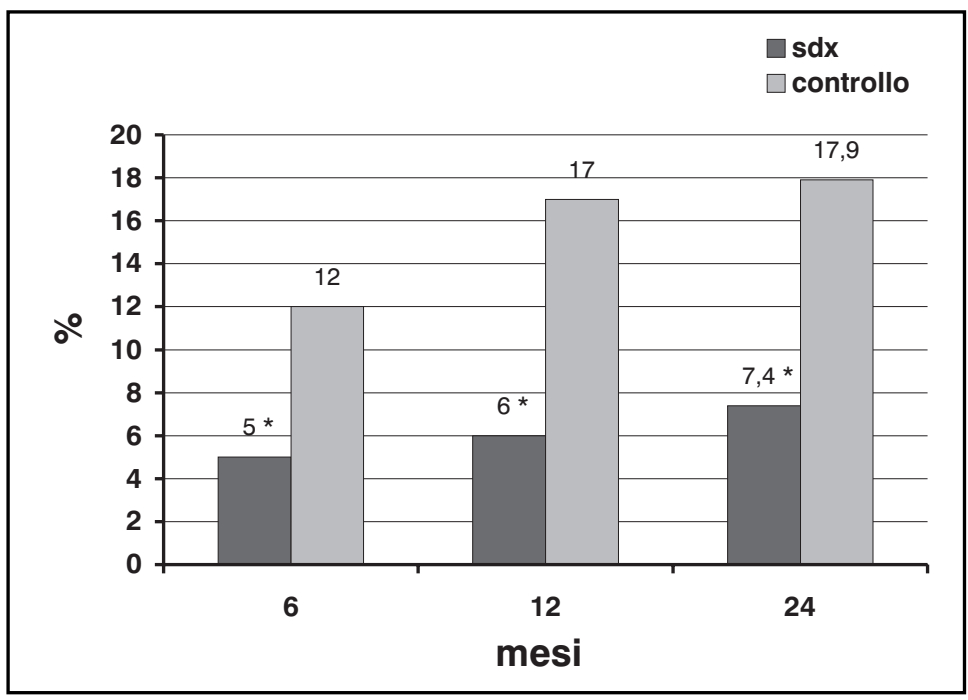

Figura 1

Incidenza di retrombosi totali nei due gruppi dopo 6,12 e 24 mesi di trattamento ${ }^{*} p<0.05$

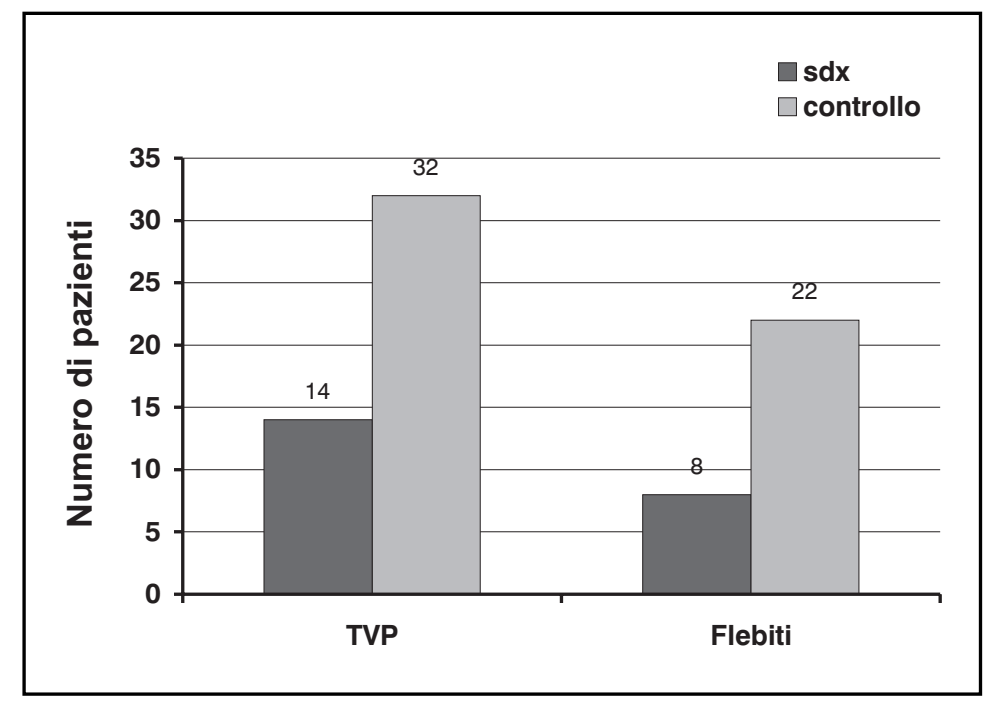

Figura 2

Incidenza di TVP e flebiti durante i 24 mesi di trattamento

Sulodexide è stato ben tollerato e la compliance è risultata elevata: il $93 \%$ dei pazienti trattati ha terminato il follow up di 24 mesi e il $97,5 \%$ delle capsule sono state assunte correttamente.

Nessuno dei casi di abbandono dello studio è stato causato dalla comparsa di effetti collaterali.

\section{Trattamento dell'arteriopatia ostruttiva periferica}

Circa il 5-6\% della popolazione con più di 65 anni di età è affetta da arteriopatia ostruttiva periferica, di cui la claudicatio intermittens rappresenta la manifestazione clinica più comune $[18,19]$. L'ostruzione arteriosa periferica avviene in seguito allo sviluppo di processi aterotrombotici localizzati a livello degli arti 


\begin{tabular}{cc}
\hline Stadio & Clinica \\
\hline Stadio I & Lesioni asintomatiche \\
Stadio II & Claudicatio intermittens \\
Ila & $150 \mathrm{~m}$ deambulazione in pianura \\
IIb & $<150 \mathrm{~m}$ deambulazione in pianura \\
Stadio III & Dolore a riposo \\
Stadio IV & Lesioni con necrosi/gangrena \\
\hline
\end{tabular}

Tabella III

Classificazione di Leriche-Fontaine delle Arteriopatie Croniche Periferiche (ACP)

inferiori; tale fenomeno può presentare diversi livelli di gravità (Tabella III) e con il peggiorare del quadro clinico aumenta notevolmente il potenziale rischio di diffusione del processo trombotico in distretti arteriosi fondamentali per la vita, come quelli coronarico e cerebrovascolare [20].

Un recente studio italiano è stato condotto su 286 pazienti con claudicatio intermittens (II stadio di Leriche-Fontaine) al fine di indagare l'efficacia di sulodexide nel trattamento della patologia ostruttiva periferica [21].

Gli effetti del farmaco sono stati valutati in base alla sua capacità di aumentare la distanza percorsa a piedi (walking performance) dei partecipanti al trial.

Gli endpoint principali dello studio riguardavano il raddoppio della distanza percorsa senza dolore e della massima distanza percorsa e la linearità fra l' evolversi di questi parametri e il tempo. Sono stati misurati inoltre l'indice pressorio caviglia-braccio e la concentrazione plasmatica di fibrinogeno; è stata valutata la gravità dei sintomi correlabili alla patologia (dolori, crampi, intorpidimenti, sensazioni di caldo/freddo all'estremità inferiori) me- diante assegnazione di un punteggio da parte del paziente.

I pazienti sono stati randomizzati a ricevere sulodexide o placebo; il farmaco è stato somministrato secondo il seguente schema posologico: $60 \mathrm{mg} / \mathrm{die}$ i.m. per i primi 20 giorni e $100 \mathrm{mg} /$ die per os per i successivi sei mesi (durata totale 27 settimane).

Riportiamo in Tabella IV i risultati ottenuti al termine dello studio nei due gruppi esaminati.

L'aumento della distanza percorsa, sia massima che senza dolore, è risultata significativamente associata al tempo di trattamento con sulodexide (analisi multifattoriale); nel caso di trattamento con placebo, tale correlazione è risultata inferiore $(\mathrm{P}=0,02$ vs. $\mathrm{P}=0,03)$.

I pazienti trattati con sulodexide hanno riportato una riduzione dello score sintomatico totale (score non riportati dagli autori dello studio).

L'analisi per sottogruppi svolta al fine di indagare l'efficacia di sulodexide in pazienti affetti da diabete di tipo II (25\% dei partecipanti) ha dimostrato risultati significativi, in linea con gli altri sottogruppi. I dati sulla sicurezza d'impiego del sulodexide indicano una riduzione nell'incidenza di gravi eventi cardiovascolari nel gruppo trattato rispetto al gruppo controllo (Tabella IV), anche se lo studio non è stato disegnato al fine di dimostrare una riduzione nell' incidenza di gravi eventi.

Dal punto di vista della tollerabilità, un totale di 58 eventi avversi è stato riportato da 44 pazienti, di cui 19 appartenenti al gruppo trattato e 25 al gruppo placebo.

Sei pazienti riceventi sulodexide e cinque riceventi placebo hanno riportato almeno un effetto indesiderato giudicato dagli autori potenzialmente correlabile all' assunzione del farmaco; per sulodexide tali effetti sono stati: diar-

\section{Tabella IV}

Risultati ottenuti nei due gruppi al termine dello studio [21]

Le percentuali sono calcolate in base alla popolazione dei pazienti intent-to-treat (ITT)

$\mathrm{Pz}=$ pazienti

\begin{tabular}{|c|c|c|c|}
\hline & Sulodexide & Placebo & $\mathbf{P}$ \\
\hline Popolazione (ITT) & 143 & 143 & \\
\hline Abbandono dello studio & $17(11,9 \%)$ & $34(23,8 \%)$ & 0,013 \\
\hline \multicolumn{4}{|c|}{ RISULTATI dopo 27 settimane di trattamento (rispetto al baseline) } \\
\hline $\begin{array}{l}\text { Pz che hanno raddoppiato la distanza percorsa } \\
\text { senza dolore }\end{array}$ & $34(23,8 \%)$ & $13(9,1 \%)$ & 0,001 \\
\hline $\begin{array}{l}\text { Aumento medio della distanza percorsa senza } \\
\text { dolore }\end{array}$ & $64,7 \%$ & $29,9 \%$ & 0,001 \\
\hline $\begin{array}{l}\text { Pz che hanno raddoppiato la distanza massima } \\
\text { percorsa }\end{array}$ & $37(25,9 \%)$ & $9(6,3 \%)$ & 0,001 \\
\hline Aumento medio della distanza massima percorsa & $76 \%$ & $27,9 \%$ & 0,001 \\
\hline $\begin{array}{l}\text { Indice pressorio caviglia-braccio medio pre- e post- } \\
\text { trattamento }\end{array}$ & $0,60 \rightarrow 0,67$ & $0,58 \rightarrow 0,62$ & - \\
\hline Variazione media concentrazione di fibrinogeno & $-31,9 \mathrm{mg} / \mathrm{dl}$ & $+30,2 \mathrm{mg} / \mathrm{dl}$ & 0,001 \\
\hline Gravi eventi cardiovascolari (num pz) & 4 & 11 & 0.040 \\
\hline
\end{tabular}


rea, dolore epigastrico, eruzioni cutanee, vertigini e dolore o ematoma in sede di iniezione.

I dati risultanti dallo studio confermano quanto rilevato da Gaddi e colleghi in una meta-analisi (19 studi esaminati) pubblicata nel 1996: sulodexide ha un'efficacia maggiore rispetto a placebo nel migliorare la walking performance, e tale differenza è statisticamente significativa [22].

\section{ASPETTI FARMACOECONOMICI}

La causa più frequente di insorgenza di ulcere degli arti inferiori è rappresentata dall'Insufficienza Venosa Cronica (70\% circa dei casi), seguita dall'insufficienza arteriosa (10\% dei casi).

L'IVC costituisce una condizione clinica assai rilevante dal punto di vista epidemiologico con importanti ripercussioni socio-economiche; nei Paesi occidentali i costi dell'iter diagnostico e terapeutico, le significative perdite in ore lavorative e le ripercussioni sulla qualità di vita collegate a questo disturbo e alle sue complicanze rappresentano un costo importante per la società [23,24].

I costi annuali per la gestione dell'IVC riportati da Ruckeley e coll. nel 1997 erano di 290 milioni di sterline per l'Inghilterra, 14,7 miliardi di franchi francesi, 2,4 miliardi di marchi tedeschi e 1.638 miliardi di lire italiane [25].

In Inghilterra e in Galles il numero di ore lavorative perse ogni anno a causa dell'insufficienza venosa cronica è pari a circa 500.000 mentre negli USA (dove 2.500.000 individui sono affetti da IVC e 500.000 da ulcere venose attive) è di 2.000.000 [23].

La prevalenza dell'IVC a carico degli arti inferiori è del 10-50\% nella popolazione adulta maschile e del $50-55 \%$ in quella femminile. $\mathrm{La}$ malattia varicosa è presente, clinicamente manifesta, nel 10-33\% delle donne e nel 10-20\% dei maschi adulti [23,26,27,28].

L'incidenza della malattia varicosa varia in funzione di numerosi fattori: l'età avanzata, il sesso femminile, il prolungato ortostatismo, la familiarità, il numero di gravidanze e l'elevato peso corporeo sembrano essere fattori predisponenti, anche se, per alcuni di essi, la letteratura non riporta dati omogenei.

\section{Ulcere Venose Croniche}

La cura delle ulcere venose costituisce un problema spesso sottovalutato e scaricato sulla famiglia del paziente. Questa patologia colpisce circa lo $0,5-3 \%$ della popolazione adulta occidentale $[29,30]$, anche se i dati epidemiologici riportati in letteratura appaio- no molto diversi a seconda delle casistiche e spesso sono poco precisi.

La guarigione avviene in tempi lunghi e la comparsa di recidive risulta molto frequente: nel 50-75\% dei casi il tessuto ripara in 6 mesi, mentre nel $20 \%$ resta aperto in media per 24 mesi e nell' $8 \%$ per 5 anni [31].

Nel lavoro svolto da Ruckeley nel 1997 è riportata una spesa annua per il management delle ulcere venose nel nostro Paese di circa 243 miliardi di lire [32].

La loro cura viene generalmente gestita dal medico curante o da un infermiere a domicilio $\mathrm{e}$, in casi più rari, presso centri specialistici. Il Lothian and Forth Valley Leg Ulcer Study ha valutato che il $71 \%$ dei pazienti con ulcere viene curato a domicilio [33].

Uno studio inglese sui costi annui per il materiale utilizzato per la medicazione delle ulcere [34] ha indicato una spesa che si aggira tra i 367 e i 733 milioni di sterline annue, circa il 2-3\% del budget sanitario nazionale.

Oltre ai costi relativi a farmaci e materiale di medicazione, la cura delle ulcere degli arti inferiori richiede un elevato consumo di risorse in termini di servizio infermieristico territoriale: la medicazione domiciliare infatti impegna circa il 10-30\% dell'orario di lavoro [35].

Nel Regno Unito il costo sociale che ne deriva si aggira intorno alle 2-4.000 sterline annue per ciascuno dei 150-200.000 pazienti con ulcere degli arti, per un totale di 600 milioni di sterline, il 2\% del budget annuale per la sanità.

In generale, secondo uno studio pubblicato nel 1994, il costo annuo per l'assistenza distrettuale di un paziente con ulcera dell' arto non complicata si aggirerebbe intorno ai $3 \mathrm{mi}-$ lioni di lire [36].

I dati risultanti da uno studio francese rilevano che un paziente con ulcera venosa in media consulta il proprio medico circa una volta al mese [37].

In Italia, secondo uno studio pubblicato nel ' 97 , si effettuano circa 291.000 visite mediche/anno per lesioni ulcerative, con prescrizioni nel $95 \%$ dei casi e un onere pari a 243 miliardi di lire [38].

L'utilizzo di un farmaco in grado di aumentare la frequenza e la velocità di guarigione delle ulcere venose e, quindi, di indurre un risparmio di risorse consumate per la loro cura rappresenta dal punto di vista economico un potenziale vantaggio. Le dimensioni di tale risparmio diventano ancora più elevate se nell'analisi dei costi, oltre alle spese più evidenti legate al materiale per medicazione, ai farmaci e al personale medico e infermieristico, vengono considerati altri oneri correlati in modo diretto o indiretto alla patologia. Fra questi ricordiamo il costo derivante dal mancato guada- 


\begin{tabular}{l} 
Categorie \\
\hline 1. Teleangectasia e/o vene superficiali leggermente dilatate o reticolate \\
2. Vene varicose senza sintomi o complicazioni \\
3. Vene varicose con principio di complicazioni \\
4. Insufficienza venosa superficiale e/o profonda con complicazioni gravi (es. \\
tromboflebiti, sanguinamenti) \\
5. Ipertensione venosa cronica e lipodermatosclerosi \\
6. Ulcerazioni iniziali di piccole dimensioni \\
7. Ulcere gravi e ricorrenti con infezione
\end{tabular}

Tabella V

Diversi quadri clinici e subclinici dei pazienti partecipanti allo studio [27]

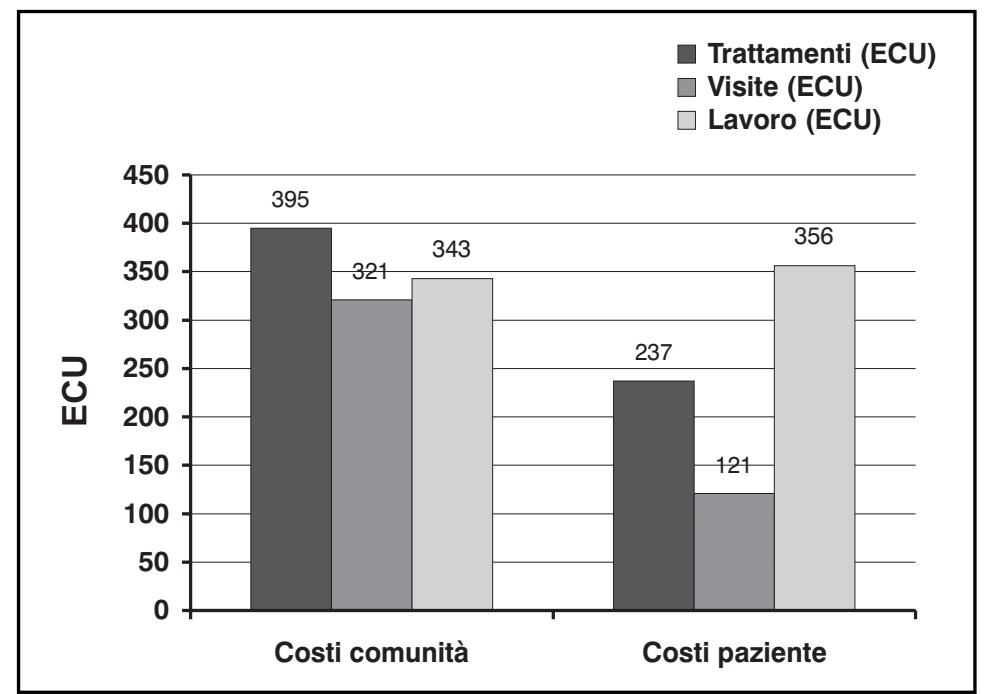

Figura 3

Spesa annuale per paziente con disturbi venosi a carico della comunità e a carico del paziente stesso suddivisa in costi per visite ed esami, per trattamento e per giorni di lavoro persi (1 ECU 1 euro)

gno per la perdita di giornate lavorative e le spese chirurgiche che possono rendersi necessarie nel caso in cui la lesione non tenda a guarire.

Secondo alcuni studi, il 12,5\% dei pazienti con ulcera in età lavorativa è costretto a ricorrere al prepensionamento [39-41].

Quando un'ulcera non guarisce mediante i comuni trattamenti, comprese medicazioni avanzate, metodiche di radiologia interventistica o procedure di rivascolarizzazione con by-pass, si ricorre solitamente alla chirurgia ricostruttiva, di cui gli innesti di cute coltivata rappresentano l'approccio più promettente; in Italia questo intervento rappresenta un costo medio per il SSN di 3.026 euro.

In casi estremi, un'ulcera non risolta può portare all' amputazione dell'arto: mediamente un'operazione di questo tipo costa al SSN circa 7.775 euro, secondo i DRG attualmente in vigore per la regione Lombardia.
Ai costi fin qui annoverati occorrerebbe aggiungere una serie di spese che spesso gravano direttamente sul paziente (il trasporto per effettuare una visita, l'acquisto di antidolorifici e di farmaci coadiuvanti, ecc.); il problema assume quindi dimensioni ancor più preoccupanti.

In Italia non esistono studi farmacoeconomici sulle ulcere venose che prendano in considerazione tutti gli aspetti qui elencati permettendo di calcolare in maniera precisa l'ammontare della spesa annua per paziente; esiste però uno studio disegnato e condotto nell' ambito del progetto "San Valentino" che ha valutato la prevalenza dei disturbi venosi all'interno di una popolazione omogenea [27].

Le caratteristiche cliniche e subcliniche, raggruppate nella definizione generica di disturbi venosi, dei pazienti considerati nello studio sono riassunte nella tabella V. Gli autori hanno registrato i costi sostenuti dal paziente e dalla comunità, suddividendoli in spese per trattamenti, per visite mediche e per giorni di lavoro persi (Figura 3).

Da questa stima i costi a carico della comunità ammonterebbero a 1.059 euro per anno, quelli del paziente a 714 euro; questi sono valori medi di pazienti con quadri clinici diversi (vedi Tabella V) e, essendo l'ulcerazione la complicanza più grave dei disturbi venosi, si desume che la spesa annuale effettivamente sostenuta dalla società per il management di un'ulcera cronica sia sensibilmente maggiore; lo stesso discorso vale per i costi sostenuti dal paziente.

Illustriamo in Tabella VI il costo del trattamento con sulodexide, quando somministrato secondo lo schema posologico utilizzato da Coccheri e coll [9].

In base ai dati risultanti dallo studio di Coccheri e coll. si può quindi affermare che con una spesa di 212,32 euro per paziente si può ottenere un aumento del $20 \%$ delle guarigioni complete dell'ulcera entro i due mesi; tale spesa risulta limitata se paragonata ai sopracitati costi sostenuti da paziente e comunità per quanto riguarda visite, giorni lavorativi persi e gestione delle complicanze.

Merita poi una particolare attenzione l'aspetto legato alla Qualità di Vita del paziente: la valutazione di una terapia delle ulcere venose non dovrebbe considerare solo il tasso di guarigione, ma tenere conto anche di altri fattori quali il controllo del dolore, delle secrezioni, la prevenzione di amputazioni e tutto ciò che riguarda la vita relazionale del paziente.

Da uno studio americano emerge che la maggior parte dei portatori di ulcera avverte sintomi di intensità media e grave di cui il dolo- 


\begin{tabular}{ccccc}
\hline Posologia & Costo confezione & Costo unitario & $\begin{array}{c}\text { Costo } \\
\text { trattamento }\end{array}$ & Costo totale \\
\hline 1fiala/die per 20 gg & 10 fiale $=22,30$ euro & 2,23 euro & 44,60 euro & \\
4 cps/die per 70 gg & $50 \mathrm{cps}=29,95$ euro & 0,599 euro & 167,72 euro & $\mathbf{2 1 2 , 3 2}$ \\
\hline
\end{tabular}

Tabella VI

Costo del trattamento con sulodexide secondo il regime posologico utilizzato nello studio condotto da Coccheri e coll. [9] re è il più frequente; 1'81\% dei pazienti dichiara che l'ulcera influisce negativamente sulla sua capacità di muoversi e di deambulare e, per i pazienti in età lavorativa, la lesione costituisce la prima causa di perdita di giorni di lavoro o di perdita del lavoro stesso. Il 58\% degli intervistati trova il management dell'ulcera molto gravoso da un punto di vista economico [42].

Uno studio inglese ha valutato la qualità di vita dei pazienti affetti da ulcere venose mediante l'utilizzo del Medical Outcomes Short Form-36. I 118 partecipanti allo studio hanno completato il questionario al baseline e dopo 12 settimane di trattamento standard dell'ulcerazione; i dati risultanti rilevano che i portatori di ulcera venosa hanno score più bassi rispetto ai valori di riferimento per quanto riguarda l'ambito emozionale $(\mathrm{P}=0,001)$, le funzioni sociali $(\mathrm{P}=0,001)$, il ruolo $(\mathrm{P}=0,001)$ e il dolore físico $(\mathrm{P}=0,001)$. Gli score migliorano leggermente al termine delle 12 settimane di trattamento, ma il dolore diminuisce solo nei pazienti in cui la lesione è guarita nel corso di tale periodo; anche l'incremento degli altri punteggi è superiore nei casi di completa guarigione.

Dai 37 studi revisionati in una review pubblicata recentemente, è emerso che le maggiori limitazioni sono dovute al dolore e all'immobilità causata dalle lesioni, aggravate da disturbi del sonno, mancanza di energia e limitazione delle attività lavorative e di svago [43].

La qualità di vita dei pazienti è risulta notevolmente peggiorata rispetto a quella di soggetti sani anche a causa della preoccupazione, della frustrazione e della mancanza di autostima derivante dalla patologia e soprattutto dalla mancanza di risultati incoraggianti in seguito alla terapia utilizzata [44].

Questo aspetto della patologia, talvolta sottovalutato, mette in luce quanto siano importanti, al fine di permettere il miglioramento della qualità di vita dei pazienti, i benefici prodotti da alcuni farmaci attivi nel velocizzare il processo di guarigione di una lesione ulcerativa.

\section{Terapia sistemica nell'Ulcera Venosa}

La terapia delle ulcere venose è basata tradizionalmente sul trattamento compressivo e sulla medicazione topica della lesione; negli ultimi anni però medici e specialisti, visti i len- tissimi tempi di riparazione e la tendenza a recidivare, hanno cominciato ad associare ai trattamenti tradizionali una terapia sistemica che agisse a livello del microcircolo riequilibrando i diversi processi biochimici che stanno alla base dello sviluppo della lesione [8].

I principali bersagli di questi farmaci sono il tono venoso, l'aumentata permeabilità capillare, I'edema, la ridotta attività fibrinolitica, l'incremento del fibrinogeno plasmatico e il controllo del dolore.

Secondo le Linee Guida Diagnostico Terapeutiche delle malattie delle vene e dei linfatici curate dal Consiglio Italiano di Flebologia, i farmaci più frequentemente impiegati sono gli agenti fibrinolitici o favorenti la fibrinolisi (ad es. il mesoglicano), gli idrossirutosidi (come l'oxerutina) [45,46], i farmaci costituiti da diosmina [32,47] e la pentossifillina [48].

A causa dei limiti metodologici di molti trial clinici l'efficacia di alcuni farmaci di supporto nella guarigione delle lesioni è ancora discussa [49]; inoltre non esistono in letteratura, se non in rarissimi casi, studi che mettano direttamente a confronto efficacia e tollerabilità dei farmaci sopracitati: non sussistono quindi le condizioni per attuare un' analisi di minimizzazione dei costi fra le diverse strategie indicate per la cura di questa patologia.

Per inquadrare e confrontare comunque la spesa farmaceutica sostenuta dal paziente per le diverse molecole utilizzate nel trattamento delle ulcere, ne riportiamo i costi/die e per ciclo terapeutico.

Tali costi (Tabelle VII, VIII, IX, X, XI ) sono stati calcolati in base alla spesa reale che il paziente deve sostenere per l'acquisto del farmaco nel corso del ciclo terapeutico (considerando il prezzo intero della confezione anche quando essa viene utilizzata solo parzialmente).

Nel computo non sono stati considerati i possibili costi di somministrazione, di management degli eventi avversi o altre spese collegate al trattamento farmacologico; non sono altresì riportate le spese relative all'acquisto di ausili o strumenti per l'elastocompressione, né di materiali per la medicazione delle lesioni ulcerative dal momento che tali spese sono comuni a tutte le terapie considerate. 
Sulodexide: implicazioni cliniche ed economiche

\section{Tabella VII}

Terapia di supporto con sulodexide nel

trattamento dell'ulcera venosa*

\section{Tabella VIII}

Terapia di supporto con mesoglicano nel trattamento dell'ulcera venosa*

\begin{tabular}{|c|c|c|c|c|}
\hline & Schema posologico & $\begin{array}{c}\text { Numero } \\
\text { confezioni/ciclo } \\
\text { terapeutico }\end{array}$ & Costo terapia & $\begin{array}{l}\text { Riferimento } \\
\text { bibliografico }\end{array}$ \\
\hline $\begin{array}{l}\text { Dose } \\
\text { minima } \\
\text { riportata in } \\
\text { letteratura }\end{array}$ & $\begin{array}{l}-60 \mathrm{mg} / \text { die i.m. per } 30 \mathrm{gg} \\
-50 \mathrm{mg} / \mathrm{die} \text { p.o. per } 30 \mathrm{gg}\end{array}$ & $\begin{array}{c}3 \text { conf. da } 10 \mathrm{f} / 60 \mathrm{mg} \\
2 \text { conf. da } 50 \mathrm{cps} / 25 \mathrm{mg}\end{array}$ & $\begin{array}{l}\text { 126,8 euro/ciclo } \\
\text { terapeutico }\end{array}$ & [50] \\
\hline $\begin{array}{l}\text { Dose } \\
\text { massima } \\
\text { riportata in } \\
\text { letteratura }\end{array}$ & $\begin{array}{l}-60 \mathrm{mg} / \mathrm{die} \text { i.m. per } 20 \mathrm{gg} \\
-100 \mathrm{mg} / \mathrm{die} \text { p.o. per } 70 \mathrm{gg}\end{array}$ & $\begin{array}{c}2 \text { conf. da10 f/60 mg } \\
6 \text { conf. da } 50 \mathrm{cps} / 25 \mathrm{mg}\end{array}$ & $\begin{array}{l}224,3 \text { euro/ciclo } \\
\text { terapeutico }\end{array}$ & [9] \\
\hline $\begin{array}{l}\text { Dosaggio } \\
\text { medio/die }\end{array}$ & 2 cps da 25 mg & - & 1,198 euro/die & $\begin{array}{l}\text { Vessel }{ }^{\circledR} \text { - } \\
\text { Riassunto } \\
\text { caratteristiche } \\
\text { del prodotto }\end{array}$ \\
\hline
\end{tabular}

* Costi reali per il paziente. I prezzi d'acquisto del farmaco sono quelli riportati in "L'Informatore Farmaceutico" - Edizione 2004.

\begin{tabular}{lcccc} 
& Schema posologico & $\begin{array}{c}\text { Numero } \\
\text { confezioni/ciclo } \\
\text { terapeutico }\end{array}$ & Costo terapia & $\begin{array}{c}\text { Riferimento } \\
\text { bibliografico }\end{array}$ \\
\hline $\begin{array}{l}\text { Dose } \\
\text { riportata in } \\
\text { letteratura }\end{array}$ & $-100 \mathrm{mg} /$ die per $21 \mathrm{settimane}$ & $\begin{array}{c}2 \mathrm{conf} \mathrm{da} 10 \mathrm{f} / 30 \mathrm{mg} \\
10 \mathrm{conf} \mathrm{da} 30 \mathrm{cps} / 50 \mathrm{mg}\end{array}$ & $\begin{array}{c}256 \mathrm{eur} / \mathrm{ciclo} \\
\text { terapeutico }\end{array}$ & {$[51]$} \\
$\begin{array}{l}\text { Dosaggio } \\
\text { medio/die }\end{array}$ & $2 \mathrm{cps}$ da $50 \mathrm{mg}$ & - & 1,52 euro/die & $\begin{array}{c}\text { Piass } \\
\text { caratteristiche } \\
\text { del prodotto }\end{array}$ \\
\hline
\end{tabular}

* Costi reali per il paziente. I prezzi d'acquisto del farmaco sono quelli riportati in "L'Informatore Farmaceutico" - Edizione 2004.

\begin{tabular}{lcccc}
\hline Schema posologico & $\begin{array}{c}\text { Numero } \\
\text { confezioni/ciclo } \\
\text { terapeutico }\end{array}$ & Costo terapia & $\begin{array}{c}\text { Riferimento } \\
\text { bibliografico }\end{array}$ \\
$\begin{array}{l}\text { Dose } \\
\text { minima } \\
\text { riportata in } \\
\text { letteratura }\end{array}$ & $1.200 \mathrm{mg} /$ die per $180 \mathrm{gg}$ & $\begin{array}{c}18 \mathrm{conf} \mathrm{da} \\
30 \mathrm{cps} / 400 \mathrm{mg}\end{array}$ & $\begin{array}{c}216 \text { euro/ciclo } \\
\text { terapeutico }\end{array}$ & {$[48,52]$} \\
$\begin{array}{l}\text { Dose } \\
\text { massima } \\
\text { riportata in } \\
\text { letteratura }\end{array}$ & $2.400 \mathrm{mg} /$ die per $180 \mathrm{gg}$ & $36 \mathrm{conf} \mathrm{da}$ & 432 euro/ciclo & \\
$\begin{array}{l}\text { Dosaggio } \\
\text { medio/die }\end{array}$ & $3 \mathrm{cps} / 400 \mathrm{mg}$ & terapeutico & {$[53]$} \\
\hline
\end{tabular}

* Costi reali per il paziente. I prezzi d'acquisto del farmaco sono quelli riportati in "L'Informatore Farmaceutico" - Edizione 2004.

\begin{tabular}{|c|c|c|c|c|}
\hline & Schema posologico & $\begin{array}{c}\text { Numero } \\
\text { confezioni/ciclo } \\
\text { terapeutico }\end{array}$ & Costo terapia & $\begin{array}{c}\text { Riferimento } \\
\text { bibliografico }\end{array}$ \\
\hline $\begin{array}{l}\text { Dose } \\
\text { minima } \\
\text { riportata in } \\
\text { letteratura }\end{array}$ & 1.000 mg/die per $90 \mathrm{gg}$ & 9 conf da $20 \mathrm{cps} / 500 \mathrm{mg}$ & $\begin{array}{c}82,80 \text { euro/ciclo } \\
\text { terapeutico }\end{array}$ & {$[54]$} \\
\hline $\begin{array}{l}\text { Dose } \\
\text { massima } \\
\text { riportata in } \\
\text { letteratura }\end{array}$ & 2.000 mg/die per 6 sett. & 9 conf da $20 \mathrm{cps} / 500 \mathrm{mg}$ & $\begin{array}{c}82,80 \text { euro/ciclo } \\
\text { terapeutico }\end{array}$ & {$[46]$} \\
\hline $\begin{array}{l}\text { Dosaggio } \\
\text { medio/die }\end{array}$ & $3 \mathrm{cps}$ da $500 \mathrm{mg}$ & - & 1,38 euro/die & $\begin{array}{c}\text { Venoruton } ® \text { - } \\
\text { Riassunto } \\
\text { caratteristiche } \\
\text { del prodotto }\end{array}$ \\
\hline
\end{tabular}

* Costi reali per il paziente. I prezzi d'acquisto del farmaco sono quelli riportati in "L'Informatore Farmaceutico" - Edizione 2004. 


\begin{tabular}{|c|c|c|c|c|}
\hline & Schema posologico & $\begin{array}{c}\text { Numero } \\
\text { confezioni/ciclo } \\
\text { terapeutico }\end{array}$ & Costo terapia & $\begin{array}{r}\text { Riferimento } \\
\text { bibliografico }\end{array}$ \\
\hline $\begin{array}{l}\text { Dose } \\
\text { minima } \\
\text { riportata in } \\
\text { letteratura }\end{array}$ & 900 mg/die per 60 gg & $\begin{array}{c}9 \text { conf da } \\
20 \mathrm{cps} / 300 \mathrm{mg}\end{array}$ & $\begin{array}{c}61,38 \\
\text { euro/ciclo } \\
\text { terapeutico }\end{array}$ & {$[55]$} \\
\hline $\begin{array}{l}\text { Dose } \\
\text { massima } \\
\text { riportata in } \\
\text { letteratura }\end{array}$ & 900 mg/die per 180 gg & $\begin{array}{c}27 \mathrm{conf} d \mathrm{da} \\
20 \mathrm{cps} / 300 \mathrm{mg}\end{array}$ & $\begin{array}{c}184,14 \\
\text { euro/ciclo } \\
\text { terapeutico }\end{array}$ & [32] \\
\hline $\begin{array}{l}\text { Dosaggio } \\
\text { medio/die }\end{array}$ & $3 \mathrm{cps}$ da $300 \mathrm{mg}$ & - & 1,024 euro/die & $\begin{array}{c}\text { Doven }{ }^{\circledR}- \\
\text { Venosmine } \\
\text { Riassunto } \\
\text { caratteristiche } \\
\text { del prodotto }\end{array}$ \\
\hline
\end{tabular}

* Costi reali per il paziente. I prezzi d'acquisto del farmaco sono quelli riportati in "L'Informatore Farmaceutico" - Edizione 2004

\section{Terapia nella Sindrome Post-Trombotica (SPT)}

Come già approfondito (vedi paragrafo "Efficacia terapeutica e tollerabilità") sulodexide è stato studiato da Errichi et al. in relazione alla sua efficacia nel prevenire la comparsa di retrombosi in pazienti con trombosi venosa profonda ad alto e medio rischio [13]. In base ai dati risultanti da questo studio gli autori hanno svolto un'analisi economica al fine di valutare l'impatto che un evento trombotico può avere sulla collettività e, soprattutto, se la prevenzione di tali eventi con sulodexide rappresenti una strategia costo/efficace.

Errichi e colleghi hanno analizzato il fatto che, nel corso dello studio, su 178 pazienti trattati con placebo si sono verificati 32 eventi trombotici, pari a 17,9 eventi su 100 pazienti in due anni e cioè circa 9 eventi all' anno. Fra 189 pazienti trattati con sulodexide, gli eventi trombotici sono stati 14; commisurato a 100 pazienti questo significa 7,4 TVP in due anni e circa 4 TVP all' anno: da questi dati appare che il trattamento di 100 pazienti con sulodexide evita circa 5 eventi trombotici all'anno.

Per quantificare il risparmio ottenuto in tal caso dalla collettività gli autori hanno utilizzato il costo medio di un evento trombotico quantificato da Cesarone e Belcaro in uno studio svolto all'interno del San Valentino Project [50]; tale costo medio è risultato pari a 23.000 euro, per un risparmio totale, dovuto alla prevenzione di 5 TVP, pari a 115.000.

Questa somma non rappresenta però un guadagno netto, in quanto per ottenere questi risultati è necessario un investimento di circa 43.200 euro per l'acquisto del farmaco per la cura di 100 pazienti.

Anche in questo caso il risparmio ottenibile mediante l'utilizzo di sulodexide come prevenzione di retrombosi può assumere dimensioni più elevate se si considera tale patologia come parziale causa di altri disturbi vascolari: nel corso del trial considerato infatti il $25 \%$ dei pazienti con recidiva aveva sviluppato una grave ipertensione venosa con il conseguente rischio di incorrere nello sviluppo di ulcerazioni.

\section{CONCLUSIONI}

Le Flebopatie rappresentano patologie ad elevato impatto socio-economico. L'evoluzione naturale di queste malattie espone infatti fasce crescenti di popolazione alle complicanze più gravi, quali le ulcere venose.

Oltre ai costi sostenuti per l'acquisto del materiale e per il personale impegnato nella medicazione delle lesioni, l'onere che grava sulla società è aumentato dai costi relativi alle ospedalizzazioni, ai trasporti, alle ore di lavoro perse e, non meno importante, alla ridotta qualità di vita dei pazienti affetti da tale patologia.

Negli ultimi anni le tradizionali strategie attuate per la cura delle ulcere, medicazione ed elastocompressione, sono state affiancate all'utilizzo di terapie farmacologiche in grado di velocizzare il processo di guarigione agendo sui diversi meccanismi fisiopatologici coinvolti.

Sulodexide è un antitrombotico eparinico indicato per il trattamento delle ulcere venose; la sua efficacia nell' aumentare la frequenza e la velocità di guarigione di tali lesioni è stata dimostrata in diversi trial; questi effetti benefici sono stati rilevati dopo due mesi e confermati dopo tre mesi di trattamento.

Sulodexide, velocizzando il processo di guarigione, contribuisce a ridurre i costi direttamente e indirettamente collegati alla patologia e quindi induce un elevato risparmio a fronte di una spesa, per l'acquisto del farmaco, limitata e in linea con le altre sostanze aventi la medesima indicazione terapeutica.
Tabella XI

Terapia di supporto con diosmina orale nel trattamento dell'ulcera venosa*

\section{NOTA BENE}

Per il calcolo del costo di terapia sono state considerate solo le formulazioni farmaceutiche a base di diosmina che sono indicate in scheda tecnica per il trattamento delle ulcere venose 
Alcuni trial hanno valutato l'efficacia clinica di sulodexide anche nel trattamento delle arteriopatie ostruttive croniche periferiche, mettendo in evidenza come tale farmaco sia in grado di migliorare la walking performance in pazienti affetti da claudicatio intermittens.

Uno studio svolto recentemente nell'ambito del San Valentino Project ha dimostrato l'efficacia del farmaco nel prevenire le recidive di trombosi; gli autori hanno rilevato che su 100 pazienti trattati, sulodexide evita in media 5 eventi trombotici ogni anno, per un risparmio netto di circa 70.000 euro a favore della società.

In tutti i trial citati il farmaco è stato ben tollerato e la compliance è risultata elevata.

In conclusione, appare chiaro come il problema delle Flebopatie abbia un notevole im- patto socio-economico; risulta perciò di fondamentale importanza eseguire un'accurata diagnosi e compiere una scelta terapeutica basata sull'evidenza scientifica. È pertanto auspicabile l'approfondimento delle conoscenze sulla cura dei disturbi venosi e del loro decorso, eventualmente mediante lo svolgimento di trial comparativi fra i diversi farmaci e l'implementazione di modelli farmacoeconomici.

Le evidenze cliniche e farmacoeconomiche di sulodexide nella terapia delle ulcere venose e nella prevenzione di alcune complicanze della Sindrome Post-Trombotica (cfr. retrombosi) rappresentano interessanti elementi da approfondire al fine di valutare la terapia con il miglior rapporto costo/efficacia per la cura e la prevenzione delle complicanze della IVC.

\section{BIBLIOGRAFIA}

1. Almond S., O'Donnel O. Cost an1. Frederick A et al. Pharmacological actions of sulodexide. Semin Thromb Hemost. 1998;24(2):127-38.

2. Harenberg J. Review of pharmacodynamics, pharmacokinetics, and therapeutic properties of sulodexide. Med Res Rev. 1998 Jan;18(1):1-20.

3. Callas DD et al. Comparative pharmacological profile of a glycosaminoglycan mixture (sulodexide) and a chemically modified heparin derivative, suleparoide. Semin Thromb Hemostas 1993; 19(suppl 1):49-57.

4. Brister SJ et al. Thrombin generation during cardiac surgery: is heparin the ideal anticoagulant? Thromb Haemost. 1993 Aug 2;70(2):259-62.

5. Vessel® - Riassunto delle caratteristiche del prodotto.

6. Porter 1995. Porter JM, Moneta G and International Consensus Committee on Chronic Venous Disease: Reporting standards in venous disease. J Vasc Surg, 1995; 2: 635-45.

7. Belcaro G. Flussimetria laser-Doppler e micro-circolazione. Ed. Minerva Medica.

8. Ennis WJ et al. Standard, Appropriate, and Advanced Care and Medical-Legal Considerations: Part TwoVenous Ulcerations (A). Wounds - ISSN: 1044-7946 - Volume 15 - Issue 4 - April 2003. 
9. Coccheri $\mathrm{S}$ et al. Randomised, double blind, multicentre, placebo controlled study of sulodexide in the treatment of venous leg ulcers. Thromb Haemost. 2002 Jun;87(6):947-52.

10 Palareti G et al. Predictive value of D-dimer test for recurrent venous thromboembolism after anticoagulation withdrawal in subjects with a previous idiopathic event and in carriers of congenital thrombophilia. Circulation. 2003 Jul 22;108(3):313-8. Epub 2003 Jul 07.

11. Pinto A et al. Sulodexide versus calcium heparin in the medium-term treatment of deep vein thrombosis of the lower limbs. Angiology. 1997 Sep;48(9):805-11.

12. Penka M. Sulodexide in the treatment of venous thrombosis. Vnitr Lek. 1995 Mar;41(3):169-71.

13. Errichi BM et al. Prevention of recurrent deep venous thrombosis with sulodexide: the SanVal registry. Angiology. 2004 May-Jun;55(3):243-9.

14. Prevention of venous thromboembolism. International Consensus Statement. Edition 2002. London: Med-Orion, 2002.

15. Hull RD et al. Different intensities of oral anticoagulant therapy in the treatment of proximal-vein thrombosis. $\mathrm{N}$ Engl J Med 1982; 307: 1676-81.

16. Kearon $\mathrm{C}$ et al. A comparison of three months of anticoagulation with extended anticoagulation for a first episode of idiopathic venous thromboembolism. N Engl J Med . 1999; 340: 901-7.

17. Linee guida per la diagnosi e il trattamento della trombosi venosa profonda. A cura di: Società Italiana di Angiologia e Patologia Vascolare, Società Italiana per lo Studio dell'Emostasi e Trombosi, Società Italiana di Diagnostica Vascolare, Collegio Italiano di Flebologia.

18. Fowkes FG et al. Edinburgh Artery Study: prevalence of asymptomatic and symptomatic peripheral arterial disease in the general population. Int J Epidemiol. 1991 Jun;20(2):384-92.

19. Dormandy J et al. Fate of the patient with chronic leg ischaemia. J Cardiovasc Surg (Torino). 1989 Jan-Feb;30(1):50-7.

20. Leng GC et al. Use of ankle brachial pressure index to predict cardiovascular events and death: a cohort study. BMJ. 1996 Dec 7;313(7070):1440-4.

21. Coccheri S et al. Sulodexide in the treatment of intermittent claudication. Results of a randomized, double-blind, multicentre, placebo-controlled study. Eur Heart J. 2002 Jul;23(13):1057-65.

22. Gaddi A et al. Meta-analysis of some results of clinical trials on sulodexide therapy in peripheral occlusive arterial disease. J Int Med Res. 1996 Sep-Oct;24(5):389-406.

23. Nicolaides AN et al. Investigation of Chronic Venous Insufficiency. A Consensus Statement. Circulation. 2000;102:e126.

24. Franks PJ et al. Quality of Life for Leg Ulcer Patients. In: Ruckley C.V, Fowkes F.G.R., Bradbury A.W. Eds. Venous Disease. London. Springer, 1999: 51-70.

25. Ruckley CV. Socioeconomic Impact of Chronic venous Insufficiency and Leg Ulcers. Angiology, 1997; 48: 67-9.

26. Callam MJ. Epidemiology of Varicose Veins. Br J Surg, 1994; 8: 167-173.

27. Cesarone MR et al. Epidemiology and Cost of Venous Diseases in Central Italy. The San Valentino Venous Disease Project. Angiology 1997; 48: 583-93.

28. Wienert V, Willer H. Epidemiologia delle malattie venose. Faenza. C.E.L.I. Ed. 1992.

29. Morison M et al. Leg Ulcers. Second ed. Mosby 1994.

30. Simka M, Majewski E. The social and economic burden of venous leg ulcers: focus on the role of micronized purified flavonoid fraction adjuvant therapy. Am J Clin Dermatol. 2003;4(8):573-81.

31. Callam MJ et al. Chronic ulcer of the leg: clinical history. BMJ. 1987;294:1389-1391.

32. Guilhou JJ et al. Efficacy of Daflon 500 in venous leg ulcer healing: a duble blind, randomized, controlled versus placebo trial in 107 patients. Angiology 1997; 48,1: 77-85.

33. Dale JJ et al. Chronic leg ulcers. The role of the district nurse. Phlebology 1985. London, Libbey, pp 621-623, 1986.

34. Wilson E. Prevention and treatment of venous leg ulcers. Health Trends, 1989. 21: 97.

35. Bosanquet N. Costs of venous ulcers: from maintenance therapy to investment programmes. Phlebology 1992; 7 (suppl.1); 44-46.

36. Appelquist $\mathbf{J}$ et al. Diabetic foot ulcers in a multi-disciplinary setting. An economic analysis of primary healing and healing with amputation. J Int Med 1994, 235: 463-71. 
37. Levy E, Levy P.Management of venous leg ulcer by French physicians, diversity and related costs: a prospective medicoeconomic observational study. J Mal Vasc. 2001 Feb;26(1):39-44.

38. Autori vari. AA. Vari. Lesioni ulcerative cutanee. Aspetto statistico. Milano. BIMS (IMS), 1997; 13: 10-7.

39. Da Silva A, Navarro MF, Batalheiro J. The importance of chronic venous insufficiency: various preliminary data on its medico-social consequences. Phlebologie. 1992;45:439-443.

40. Nelzen O. How Can We Improve Outcomes for Leg Ulcer Patients ? In: Ruckley CV, Fowkes FGR, Bradbury AW eds: Venous disease. London Springer, 1999: 246-53.

41. Baccaglini U et al. Ètude multicentrique des ulcères veineux en Italie. Phlébologie Ann Vascul, 1997; 3: 371 -8.

42. Phillips T et al. A study of the impact of leg ulcers on quality of life: financial, social, and psychologic implications. J Am Acad Dermatol. 1994 Jul;31(1):49-53.

43. Persoon A et al. Leg ulcers: a review of their impact on daily life. J Clin Nurs. 2004 Mar;13(3):341-54.

44. Liew IH, Law KA, Sinha SN. Do leg ulcer clinics improve patients' quality of life? J Wound Care. 2000 Oct;9(9):423-6.

45. Mann RJ. A double blind trial of oral O(b-hydroxyethyl)-rutosides for stasis leg ulcers. The British Journal of Clinical Practice, 1981; 35: 79-81.

46. Stegmann $\mathrm{W}$ et al. Efficacy of $O($ b-hydroxyetyl)-rutosides in the treatment of venous varicose ulcer. Therapiewoche, 1986; 36: 1828-1833.

47. Glinsky 1999. Glinsky W et al. The beneficial augmentative effect of Micronised purified Flavonoid Fraction (MPFF) on the healing of leg ulcers: an open, multicentre controlled, randomised study. Phlebology,1999; 14: 151-7.

48. Dormandy JA. Pharmacologic treatment of venous leg ulcers. J CV Pharm 1995; 25(suppl):161-5.

49. Colgan MPet al. New approaches in the medical management of venous ulceration. Angiology. 1993 Feb;44(2):138-42.

50. Scondotto G et al. Treatment of venous leg ulcers with sulodexide. Angiology. 1999 Nov;50(11):883-9.

51. Arosio E et al. A placebo-controlled, double-blind study of mesoglycan in the treatment of chronic venous ulcers. Eur J Vasc Endovasc Surg. 2001 Oct;22(4):365-72.

52. De Sanctis MT et al. Treatment of venous ulcers with pentoxifylline: a 12-month, double-blind, placebo controlled trial. Microcirculation and healing. Angiology. 2002 Jan-Feb;53 Suppl 1:S49-51.

53. Falanga V et al. Systemic treatment of venous leg ulcers with high doses of pentoxifylline: efficacy in a randomized, placebo-controlled trial. Wound Repair Regen. 1999 Jul-Aug;7(4):208-13.

54. Wright DD et al. Oxerutins in the prevention of recurrence in chronic venous ulceration: randomized controlled trial. Br J Surg. 1991 Oct;78(10):1269-70.

55. Roztocil K, Stvrtinova V, Strejcek J. Efficacy of a 6-month treatment with Daflon 500 mg in patients with venous leg ulcers associated with chronic venous insufficiency. Int Angiol. 2003 Mar;22(1):24-31.

56. Cesarone MR, Belcaro G. San Valentino Epidemiological/Costing Study. Pescara: Cardiovascular Research Publications, 2002. 


\section{NORME REDAZIONALI}

Ogni articolo proposto per la pubblicazione su "Farmeconomia" è costituito da un testo di non più di 6.000 parole (escluse referenze e tabelle). Per ciascun contributo è necessario inviare presso l'editore (via Passalacqua, 10 - 10122 TORINO):

una stampa su carta in duplice copia del testo; una stampa su carta in duplice copia di eventuali tabelle, grafici e immagini (una per pagina); un dischetto da 3.5 pollici contenente il file di testo e di eventuali tabelle, grafici e immagini.

I permessi a riprodurre materiale precedentemente pubblicato o ad utilizzare illustrazioni che possano identificare soggetti umani.

I file devono essere preparati in ambiente DOS/ Windows. Si consiglia vivamente di usare i seguenti programmi:

- file di testo: Word.

- tabelle : Word, Excel

- grafici: Excel, Power Point

- immagini: formato TIFF, PCX, BMP, GIFF o JPEG.

Il file di testo deve essere salvato su dischetto in tre formati differenti: formato dell'applicazione originale, formato Word 6 o versione precedente (consigliabile Word 2 per Windows), formato testo (TXT).

L'articolo deve essere presentato come segue:

1. Il testo è composto in corpo $12 \mathrm{e}$ interlinea $1 \mathrm{su}$ pagine di formato $\mathrm{A} 4$. In ciascuna pagina il rettangolo di stampa ha dimensioni di $24 \mathrm{~cm}$ (altezza) per 15 $\mathrm{cm}$ (larghezza).

Le illustrazioni non possono avere dimensioni maggiori di $203 \times 254 \mathrm{~mm}$

2. Il testo è strutturato secondo le seguenti sezioni:

pagina 1:

a.- Titolo;

b.- Una interlinea senza testo;

c.- Autori (indicando per ognuno il nome per esteso e il più alto titolo accademico) ;

d.- Una interlinea senza testo;

e.- Istituzioni di appartenenza;

f.- Una interlinea senza testo;

g.- nome, indirizzo, telefono dell'autore responsabile della corrispondenza riguardo l'articolo in questione.

pagina 2

h.- La parola INTRODUZIONE;

i.- Una sezione di testo che illustra gli obiettivi del lavoro;

nuova pagina

j.- Le parole MATERIALI E METODI; k.- Una sezione di testo che illustra i materiali e i metodi del lavoro;

nuova pagina

1.- La parola RISULTATI;

m.- Una sezione di testo che presenta i risultati; nuova pagina

n.- La parola DISCUSSIONE;

o.- Una sezione di testo riportante la discussione del lavoro;

nuova pagina

p.- La parola BIBLIOGRAFIA;

q.- La bibliografia del lavoro organizzata per ordine di citazione;

nuova pagina

r.- Un abstract di 300 parole circa in inglese

La bibliografia è organizzata secondo le regole del "New England Journal of Medicine"; pertanto la citazione dei riferimenti all'interno del testo è fatta inserendo i rispettivi numeri come apici.

Eventuali tabelle e figure (identificate da numeri arabi) sono tutte corredate da una legenda sintetica che ne riassume il contenuto. Le pagine debbono essere numerate progressivamente scrivendo il numero nella parte inferiore del foglio. Lo stampato deve corrispondere esattamente ai file riportati su dischetto.

All'atto dell'invio dell' articolo l'autore deve fornire una lista di tutti gli articoli di argomento simile che sono stati precedentemente inviati ad altre testate e che quindi si possano considerare come duplicati o ridondanti dello stesso lavoro. L'autore deve avvertire l'editore se il lavoro include soggetti su cui è stato pubblicato un precedente rapporto. Ognuno di questi lavori deve essere riportato nella bibliografia. Copia di questo materiale deve essere inviata all'editore.

La riedizione di uno stesso articolo deve essere approvata dall'editore che per primo l'ha pubblicato e deve essere indirizzato ad un target di lettori differente, oltre, possibilmente, ad esser stato pubblicato in altra lingua. In questo caso si deve allegare la seguente nota: "Questo articolo è basato su studi precedentemente riportati su [nome del giornale con i riferimenti completi].

\section{PROTEZIONE DEL DIRITTO ALLA PRIVACY}

Nel caso di articoli in cui vi siano studi su pazienti, questi godono del diritto alla privacy che non va infranta senza il consenso informato. Informazioni sull'identità non possono essere pubblicate ne in forma di descrizioni scritte ne fotografiche o sotto altra forma a meno che non siano essenziali per gli scopi scientifici e il paziente abbia rilasciato consenso informato in forma scritta. I dati dei pazienti non possono in alcun caso essere alterati o falsificati al fine di ottenere l'anonimato. 


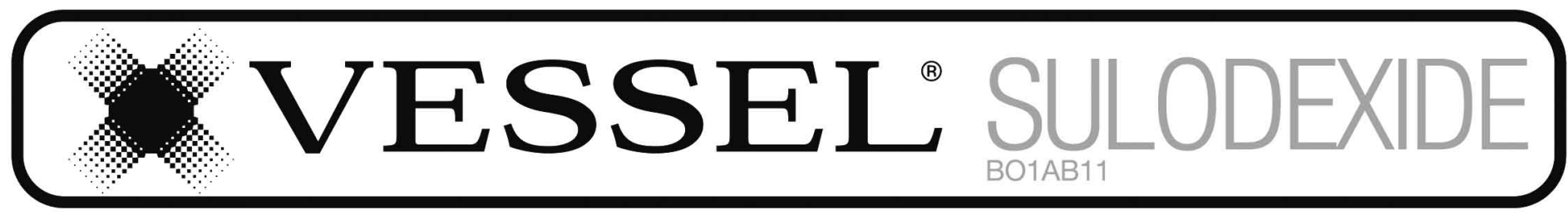

\section{RIASSUNTO DELLE CARATTERISTICHE DEL PRODOTTO}

1. DENOMINAZIONE DEL MEDICINALE:

VESSEL ${ }^{B} 250$ ULS CAPSULE MOLLI

VESSEL ${ }^{\oplus} 600$ ULS/2 ML SOLUZIONE INIETTABILE

\section{COMPOSIZIONE QUALITATIVA E QUANTITATIVA:}

Capsule molli: Sulodexide ULS 250

Fiale: Sulodexide ULS 600

Per gli eccipienti, vedere 6.1

\section{FORMA FARMACEUTICA}

Capsule molli

Soluzione iniettabile per somministrazione intramuscolare ed endovenosa

\section{INFORMAZIONI CLINICHE:}

4.1 Indicazioni terapeutiche:

Ulcere venose croniche.

4.2 Posologia e modo di somministrazione:

VESSEL ${ }^{\circ} 250$ ULS CAPSULE MOLLI: 1 capsula 2 volte al di, Iontano dai pasti.

VESSEL ${ }^{\circledR} 600$ ULS/2 ML SOLUZIONE INIETTABILE: 1 fiala al dì, per via i.m. od e.v.

Orientativamente si consiglia di iniziare la terapia con le fiale e, dopo 15-20 giorni, proseguire con le capsule per $30-40$ giorni. II ciclo terapeutico completo va ripetuto almeno due volte l'anno.

A giudizio del medico, la posologia può essere variata in quantità e frequenza.

4.3 Controindicazioni:

Ipersensibilità al principio attivo o ad uno qualsiasi degli eccipienti, verso l'eparina e gli eparinoidi. Diatesi e malattie emorragiche.

4.4 Avvertenze speciali e opportune precauzioni d'impiego:

VESSEL, per le sue caratteristiche farmaco-tossicologiche, non presenta particolari precauzioni d'uso. Comunque, nei casi in cui sia anche in atto un trattamento con anticoagulanti, è consiglia-bile controllare periodicamente i parametri emocoagulativi.

Tenere fuori dalla portata dei bambini.

4.5 Interazioni con altri medicinali e altre forme di interazione:

Essendo Sulodexide una molecola eparino-simile può aumentare gli effetti anticoagulanti dell'eparina stessa e degli anticoagulanti orali se somministrato contemporaneamente.

4.6 Gravidanza e allattamento:

Per motivi cautelativi, se ne sconsiglia l'uso in gravidanza, anche se gli studi di tossicità fetale non hanno messo in evidenza effetti embrio-feto-tossici.

4.7 Effetti sulla capacità di guidare veicoli e sull'uso di macchinari:

VESSEL non influisce o influisce in modo trascurabile sulla capacità di guidare veicoli o di usare macchinari.

4.8 Effetti indesiderati:

Segnalati occasionalmente:

Capsule molli: disturbi dell'apparato gastroenterico con nausea, vomito ed epigastralgie.

Fiale: dolore, bruciore ed ematoma in sede di iniezione.

Inoltre, in rari casi, si può avere sensibilizzazione con manifestazioni cutanee 0 in sedi diverse

4.9 Sovradosaggio:

L'incidente emorragico è l'unico effetto ottenibile da un sovradosaggio. In caso di emorragia occorre iniettare, come si usa nelle 'emorragie epariniche', solfato di Protamina all' $1 \%$ ( $3 \mathrm{ml}$ i.v. $=30 \mathrm{mg}$ ).

\section{PROPRIETÀ FARMACOLOGICHE:}

L'attività del Sulodexide si esplica mediante una spiccata azione antitrombotica sia sul versante arterioso che venoso.

5.1 Proprietà farmacodinamiche:

Categoria farmacoterapeutica: Sulodexide è classificato tra i farmaci antitrombotici eparinici Codice ATC: B01AB11.

\section{Meccanismo d'azione:}

Numerosi studi clinici condotti somministrando il prodotto per via parenterale ed orale, dimostrano che l'attività antitrombotica del Sulodexide è dovuta all'inibizione dose-dipendente di alcun fattori coagulativi tra cui, in primo luogo, il fattore Xattivato, mentre l'interferenza con la trombina, restando a livelli poco significativi, evita in genere le conseguenze di una azione anticoagulante.

L'azione antitrombotica è sostenuta anche dall'inibizione della adesività piastrinica e dall'attivazione del sistema fibrinolitico circolante e di parete.

II Sulodexide, inoltre, normalizza i parametri viscosimetrici che di solito si ritrovano alterati in pazienti con patologie vascolari a rischio trombotico: tale attività si esercita principalmente mediante la riduzione dei valori di fibrinogeno.

II profilo farmacologico sin qui descritto per Sulodexide, è completato dalla normalizzazione de valori lipidici alterati, ottenuta mediante attivazione della lipoproteinlipasi.

Effetti farmacodinamici: studi volti ad evidenziare eventuali altri effetti, oltre a quelli sopra descritti, che sono alla base dell'efficacia terapeutica, hanno permesso di confermare che la somministrazione di VESSEL non mostra effetti anticoagulanti.

\subsection{Proprietà farmacocinetiche:}

a) caratteristiche generali del principio attivo

Sulodexide presenta un assorbimento attraverso la barriera gastrointestinale dimostrabile in base agli effetti farmacodinamici dopo somministrazione per via orale, intraduodenale, intrailea- le e rettale nel ratto di Sulodexide marcato con fluoresceina. Sono state dimostrate le correlazioni dose-effetto e dose-tempo nel ratto e nel coniglio previa somministrazione per le vie sopraelencate. La sostanza marcata si accumula inizialmente nelle cellule dell'intestino per po essere liberata dal polo sierico nel circolo sistemico. La concentrazione della sostanza radioattiva aumenta nel tempo significativamente a livello di cervello, rene, cuore, fegato, polmone testicolo, plasma.

Prove farmacologiche eseguite nell'uomo con somministrazioni i.m. e i.v. hanno dimostrato relazioni lineari dose-effetto. II metabolismo è risultato principalmente epatico e l'escrezione principalmente urinaria.

L'assorbimento dopo somministrazione orale nell'uomo, studiato con il prodotto marcato, ha evidenziato che un primo picco ematico si determina alle 2 ore ed un secondo picco tra la quarta e la sesta ora, dopo di che il farmaco non è più determinabile nel plasma e ricompare verso la dodicesima ora, rimanendo quindi costante fin verso la quarantottesima ora. Questo costante valore ematico riscontrato dopo la dodicesima ora è probabilmente dovuto al lento rilascio de farmaco da parte degli organi di captazione ed in particolare dell'endotelio dei vasi. Escrezione urinaria: utilizzando il prodotto marcato, si è registrata una escrezione urinaria media del 55,23\% della radioattività somministrata, nell'arco delle prime 96 ore. Tale eliminazione mostra un picco attorno alle 12 ore, con un valore medio urinario, nell'intervallo $0-24$ ore, del $17,6 \%$ della dose somministrata; un secondo picco attorno alla $36 \mathrm{ma}$ ora, con eliminazione urinaria tra le 24-48 ore del $22 \%$ della dose; un terzo picco attorno alla 78 ma ora con un'eliminazione di circa il $14,9 \%$ nel periodo $48-96$ ore. Dopo 96 ore non è più rilevabile la radioattività nei campioni raccolti. Escrezione fecale: la radioattività totale recuperata nelle feci è del $23 \%$ nelle prime 48 ore dopo di che non è più rilevabile la sostanza marcata.

b) caratteristiche di particolare interesse per il paziente

L'attività terapeutica di VESSEL è stata sempre valutata in pazienti affetti da patologie vascola ri con rischio trombotico, sia sul versante arterioso che venoso.

II farmaco ha dimostrato particolare efficacia in pazienti anziani ed in pazienti diabetici.

5.3 Dati preclinici di sicurezza:

- Tossicità acuta: somministrato nel topo e nel ratto, non provoca alcuna sintomatologia tossica sino alle dosi di $240 \mathrm{mg} / \mathrm{kg}$ per os; la DL50 nel topo è di >9000 mg/kg/os e $1980 \mathrm{mg} / \mathrm{kg} / \mathrm{i}$.p.; nel ratto la DL50 è sempre $>9000 \mathrm{mg} / \mathrm{kg} / \mathrm{os}$ e $2385 \mathrm{mg} / \mathrm{kg} / \mathrm{i} . \mathrm{p}$

- Tossicità subacuta: somministrato per 21 giorni os alla dose di $10 \mathrm{mg} / \mathrm{kg}$ nel cane, non ha dato luogo a fenomeni di intolleranza, a variazioni dei parametri ematochimici ed a modificazion anatomo-patologiche dei principali organi.

- Tossicità cronica: somministrato per os per 180 giorni alla dose di $20 \mathrm{mg} / \mathrm{kg}$ nel ratto e ne cane, non ha presentato al termine del trattamento alcuna variazione di rilievo del quadro ematologico, dei parametri urinari e fecali e dei parametri istologici a carico dei principali organi.

- Tossicità fetale: alle prove di tossicità fetale nel ratto e nel coniglio ( $25 \mathrm{mg} / \mathrm{kg}$ per os) è risultato privo di effetti embrio-feto-tossici.

- Mutagenesi: risulta sprovvisto di attività mutagena nei seguenti tests: Ames; sintesi riparativa non programmata di DNA in linfociti umani (UDS); non disgiunzione in Aspergillus; crossing over in Aspergillus; soppressori di metionina in Aspergillus.

\section{INFORMAZIONI FARMACEUTICHE:}

\subsection{Elenco degli eccipienti}

\section{VESSE ${ }^{\circledR} 250$ UL S CAPSULE MOLL}

Sodio laurilsarcosinato, silice precipitata, trigliceridi, gelatina, glicerolo, sodio p-ossibenzoato di etile, sodio p-ossibenzoato di propile, biossido di titanio $E 171$, ossido di ferro rosso $E 172$ VESSEL ${ }^{\circledR} 600$ ULS/2 ML SOLUZIONE INIETTABILE

Sodio cloruro, acqua per preparazioni iniettabili.

6.2 Incompatibilità:

Sulodexide, essendo un polisaccaride acido, se somministrato in associazioni estemporanee può reagire complessandosi con tutte le sostanze basiche. Le sostanze in uso comune incompatibili nelle associazioni estemporanee per fleboclisi, sono: vitamina $\mathrm{K}$, vitamine del complesso $\mathrm{B}$, idrocortisone, jaluronidasi, gluconato di calcio, sali di ammonio quaternario, cloramfenicolo, tetracicline, streptomicina.

6.3 Periodo di validità:

A confezionamento integro: 5 anni.

6.4 Speciali precauzioni per la conservazione:

Nessuna speciale precauzione per la conservazione

6.5 Natura e contenuto del contenitore:

VESSEL ${ }^{\oplus} 250$ ULS CAPSULE MOLLI: Astuccio di cartone contenente 2 blister da 25 capsule molli cadauno.

VESSEL ${ }^{\oplus} 600$ ULS/2 ML SOLUZIONE INIETTABILE: Astuccio di cartone contenente vaschetta di polistirolo da 10 fiale di soluzione iniettabile in vetro scuro.

6.6 Istruzioni per l'uso:

Nessuna istruzione particolare

7. TITOLARE DELL'AUTORIZZAZIONE ALL'IMMISSIONE IN COMMERCIO:

ALFA WASSERMANN S.p.A.

Sede legale: Contrada S. Emidio, s.n.c. - 65020 ALANNO (Pescara)

Sede amministrativa: Via Ragazzi del '99, 5 - 40133 BOLOGNA

8. NUMERO(I) DELL'AUTORIZZAZIONE (DELLE AUTORIZZAZIONI) ALL'IMMISSIONE IN COMMERCIO:

50 capsule molli 250 ULS: A.I.C. $n^{\circ} 022629113$

10 fiale 600 ULS: A.I.C. $n^{\circ} 022629101$

9. DATA DELLA PRIMA AUTORIZZAZIONE/RINNOVO DELL'AUTORIZZAZIONE: 24/02/1982 - 01/06/2000.

10. DATA DI REVISIONE DEL TESTO: Decreto № 339 del 23/7/2003 